\title{
Hydrogen-Saturated Silicon Nanowires Heavily Doped with Interstitial and Substitutional Transition Metals
}

\author{
E. Durgun, ${ }^{* \dagger, \dagger}$ D. I. Bilc, ${ }^{\dagger, \S}$ S. Ciraci, ${ }^{\|, \perp}$ and Ph. Ghosez ${ }^{\dagger}$ \\ ${ }^{\dagger}$ Physique Theorique des Matériaux, Université de Liège (B5), B-4000 Liège, Belgium \\ ${ }^{*}$ Department of Materials Science and Engineering, Massachusetts Institute of Technology, Cambridge, Massachusetts 02139, United \\ States \\ ${ }^{\S}$ Mol \& Biomol Phys Dept, Natl Inst Res \& Dev Isotop \& Mol Technol, RO-400293 Cluj Napoca, Romania \\ ${ }^{\|}$Department of Physics and ${ }^{\perp}$ UNAM-National Nanotechnology Research Center and Institute of Materials Science and \\ Nanotechnology, Bilkent University, Bilkent, Ankara 06800, Turkey
}

ABSTRACT: We report a first-principles systematic study of atomic, electronic, and magnetic properties of hydrogen-saturated silicon nanowires ( $\mathrm{H}-\mathrm{SiNW}$ ) that are heavily doped by transition metal (TM) atoms placed at various interstitial and substitutional sites. Our results obtained within the conventional GGA+U approach have been confirmed using a hybrid functional. To reveal the surface effects, we examined three different possible facets of $\mathrm{H}$ SiNW along the [001] direction with a diameter of $\sim 2 \mathrm{~nm}$. The energetics of doping and resulting electronic and magnetic
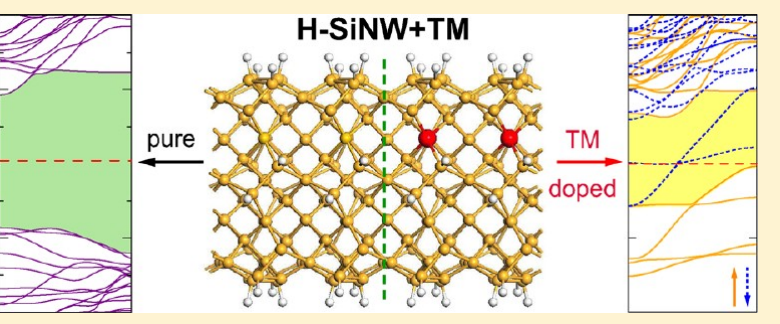
properties are examined for all alternative configurations. We found that except $\mathrm{Ti}$, the resulting systems have a magnetic ground state with a varying magnetic moment. Whereas $\mathrm{H}$-SiNWs are initially nonmagnetic semiconductor, they generally become ferromagnetic metal upon TM doping. They can even exhibit half-metallic behavior for specific cases. Our results suggest that $\mathrm{H}$-SiNWs functionalized by TM impurities form a new type of dilute magnetic semiconductor potentially attractive for new electronic and spintronic devices on the nanoscale.

\section{INTRODUCTION}

Among the research for building blocks in nanodevices, silicon nanowires ( $\mathrm{SiNWs}$ ) are attracting increasing interest due to their remarkable physical, electronic, thermal, and chemical properties. $^{1-7}$ Currently, rodlike, oxidation-resistant SiNWs can be fabricated with a diameter down to $1 \mathrm{~nm}$, where quantum confinement effects are also observable. ${ }^{5,8}$ The compatibility with current silicon-based technology ${ }^{9,10}$ makes SiNWs even more attractive, and they can enter various device applications such as field effect transistors, ${ }^{11}$ light-emitting diodes, ${ }^{12}$ lasers, ${ }^{13}$ nanosensors, ${ }^{14}$ and so on. The advances in synthesis will possibly increase the number of potential applications in various other fields.

At the theoretical level, bare and hydrogen-saturated silicon nanowires (H-SiNWs) have been extensively analyzed by using first-principles calculations. ${ }^{15-21}$ It is shown that whereas bare SiNWs are in general metallic, they become semiconductor (insulator) when saturated with hydrogen..$^{15,20,21}$ The electronic band gap can be engineered by varying diameter, growth direction, and cross section due to the quantum confinement effects. ${ }^{8,15}$ More recently, superlattice structures leading to confined states induced by diameter-modulated $\mathrm{SiNWs}^{22}$ and merged $\mathrm{Si}$ and Ge nanowires ${ }^{23}$ have been predicted.

Furthermore, growing research interest has also been devoted to functionalization of SiNWs with various dopants to study the chemical and biological sensitivities. ${ }^{14,24,25}$ The nand $\mathrm{p}$-doped $\mathrm{H}$-SiNW can provide excess carriers required in device applications like diodes and transistors. ${ }^{26-28}$ Recently, $\mathrm{Wu}$ et al. ${ }^{29}$ have reported the room-temperature magnetism of Mn-implanted SiNWs, which opens the field to spintronic applications through transition-metal (TM) doping. Theoretically, $\mathrm{Mn}$ impurities in [111] $\mathrm{SiNWs}^{30}$ and also external adsorption of various TM atoms on H-SiNWs are reported. ${ }^{20,21}$

In this article, we present an extensive first-principles study on hydrogen-saturated silicon nanowires that are heavily doped by transition metal atoms ( $\mathrm{Ti}, \mathrm{V}, \mathrm{Cr}, \mathrm{Mn}, \mathrm{Fe}$, and $\mathrm{Co}$ ) placed at various interstitial and substitutional sites aiming to obtain a new type of dilute magnetic semiconductor that would lead to new electronic and spintronic devices on the nanoscale. The energetics of doping and resulting electronic and magnetic properties are examined for all alternative configurations. To reveal surface effects, three different facets along the [001] direction are considered. The magnetic ground state is determined by considering nonmagnetic, ferromagnetic, and antiferromagnetic configurations. We also considered calculations by using a new type of hybrid functionals, which in turn are compared with the results obtained by GGA+U.

Received: April 2, 2012

Revised: June 18, 2012

Published: July 12, 2012 


\section{METHODOLOGY}

We have performed first-principles plane wave calculations within density functional theory (DFT) ${ }^{31}$ implemented in VASP code. $^{32}$ All calculations for nonmagnetic, ferromagnetic, and antiferromagnetic states are carried out by using the projectoraugmented wave (PAW) potentials. ${ }^{33,34}$ The exchange correlation potential has been approximated by generalized gradient approximation (GGA). ${ }^{35}$

To model our silicon nanowires, we have used a supercell approach, including a $10 \AA$ vacuum space between wire replica along the two directions perpendicular to the wire axis. For doped nanowires, the impurity TM atom is periodically repeated along the [001] direction corresponding to the wire axis. In most calculations, one TM atom is introduced per basic wire unit, corresponding to a fixed dopant concentration of 1.5 to $2 \%$ (depending on the facet) and a distance between consecutive impurities of $5.4 \AA$. In a later section, we further discuss the dependency in terms of dopant concentration and size.

In the self-consistent potential and total energy calculations, the Brillouin zone of supercell is sampled in the $\mathbf{k}$-space within the Monkhorst-Pack scheme ${ }^{36}$ by $(1 \times 1 \times 15)$ mesh points. A plane-wave basis set with a kinetic energy of $500 \mathrm{eV}$ has been used. All atomic positions and lattice constants along the wire axis are optimized by using the conjugate gradient method, where total energy and atomic forces are minimized. The convergence in energy is chosen as $10^{-5} \mathrm{eV}$ between two ionic steps, and the maximum force allowed on each atom is 0.01 $\mathrm{eV} / \AA$.

To validate our conclusions, some additional calculations have also been performed using the GGA+U approach of Dudarev, ${ }^{37}$ the HSE hybrid functional, ${ }^{38}$ and the so-called B1WC hybrid functional, ${ }^{39}$ which mixes $16 \%$ of Hartree-Fock exchange with $\mathrm{Wu}-$ Cohen $\mathrm{GGA}^{40}$ within $\mathrm{B} 1$ scheme. ${ }^{41}$ The B1WC hybrid functional calculations have been performed using the linear combination of atomic orbitals method, as implemented in the CRYSTAL code. ${ }^{42}$ The B1-WC hybrid functional used in this work was previously developed, ${ }^{39}$ and it was tested for H-SiNWs. ${ }^{43}$ We used localized Gaussian-type basis sets including polarization orbitals and considered all the electrons for $\mathrm{Si}^{44} \mathrm{H}^{45} \mathrm{Cr},{ }^{46}$ and $\mathrm{Fe}^{47}$ The Brillouin zone integrations were performed using a $1 \times 1 \times 15$ mesh of $\mathrm{k}$ points, and a secondary $2 \times 2 \times 30$ Gilat k-mesh was used in the calculation of Fermi energy and density matrix. The selfconsistent-field calculations were converged until the energy changes between interactions were smaller than $10^{-8}$ Hartree using a Fermi smearing of 0.00032 hartree $(\sim 100 \mathrm{~K})$. An extralarge predefined pruned grid consisting of 75 radial points and 974 angular points was used for the numerical integration of charge density. The level of accuracy in evaluating the Coulomb and exchange series is controlled by five parameters: ${ }^{42}$ the values used in our calculations are 7, 7, 7, 7, and 14. The B1WC calculations have been performed for the GGA-optimized structures obtained with the vaSP code.

\section{HYDROGEN-SATURATED SILICON NANOWIRES}

Before considering interstitial TM doping, we studied structural and electronic properties of prototype bare $[\operatorname{SiNW}(N)]$ and hydrogen-saturated $[\mathrm{H}-\mathrm{SiNW}(N)]$ silicon nanowires for $N=$ 45,57 , and 61 , where $N$ is the number of silicon atoms in the unit cell of nanowires (Figure 1). These H-SiNWs correspond to three possible facets along the [001] direction, and their
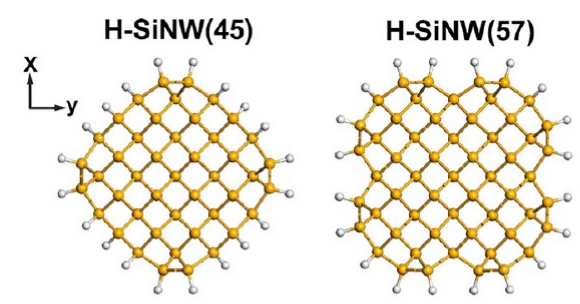

H-SiNW(61)
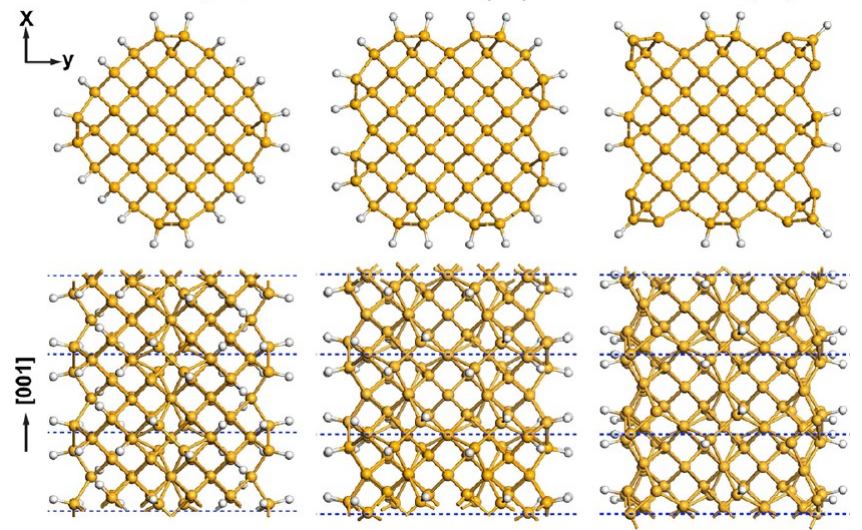

Figure 1. Top and side view of hydrogen-saturated silicon nanowires along the [001] direction with different facets for $N=45,57$, and 61, where $N$ corresponds to the number of silicon atoms in the unitcell of nanowire.

diameter changes between 1.5 and $2.1 \mathrm{~nm}^{48}$ As already discussed in the Introduction, $\mathrm{H}$-SiNWs as small as $1 \mathrm{~nm}$ have been fabricated, ${ }^{8}$ and at such small sizes edge effects become more important. ${ }^{48,49}$

The initial atomic positions of SiNWs are taken from silicon bulk crystal. Depending on the cross-section, three alternative facets are possible along [001] direction. Upon ionic relaxation by minimizing both the total energy and forces on the atoms, the ground-state configurations are obtained. The cohesive energy $\left(E_{\mathrm{C}}\right)$ of the SiNWs is calculated by the definition $E_{\mathrm{C}}=$ $E_{\mathrm{T}}[\mathrm{Si}]-E_{\mathrm{T}}[\operatorname{SiNW}(N) / N]$, where $E_{\mathrm{T}}[\mathrm{Si}]$ and $E_{\mathrm{T}}[\operatorname{SiNW}(N)]$ are the total energies of free silicon atom and $\operatorname{SiNW}(N)$, respectively. $E_{\mathrm{C}}$ is calculated to be $4.91,4.97$, and $4.99 \mathrm{eV}$ for $N$ $=45,57$, and 61, respectively. Interestingly, $\operatorname{SiNW}(61)$, which has sharp corners, has the highest $E_{\mathrm{C}}$, which is in agreement with the results of $\mathrm{Cao}$ and coworkers. ${ }^{48}$ Clearly, the presence of edges has a profound effect on the surface reconstruction of $\mathrm{SiNW}$ and thereby its electronic structure and stability. ${ }^{48,49}$ Accordingly, whereas thick SiNWs prefer cylindrical or prism shape with a core that preserves diamond structure, the cross section with sharp corners becomes more favorable for very thin nanowires. ${ }^{48}$

As a next step, we saturate the dangling bonds of silicon atoms on the surface with hydrogen ${ }^{8,20}$ and fully relax the structure. It is observed that while SiNWs are all metallic due to the surface states, they become semiconductors upon hydrogen saturation as shown in Figure 2. The band gap $\left(E_{\mathrm{G}}\right)$ tends to decrease from $N=45\left(E_{\mathrm{G}}=1.79 \mathrm{eV}\right)$ to $N=61\left(E_{\mathrm{G}}=1.16 \mathrm{eV}\right)$ due to an increase in diameter (despite the cross sections are different). This is consistent with predicted quantum confinement effects.

It is well known that whereas calculations based on DFT typically yield very accurate structural properties for silicon and other semiconductors the standard approximations (LDA or GGA) lead to a significant underestimation of the electronic band gaps due to intrinsic failure in handling self-interactions. ${ }^{50}$ Accordingly, we recalculated the band gaps by using B1-WC hybrid functional, ${ }^{39}$ the details of which are given in the Methodology section. We noticed that the band structure profiles that are obtained by GGA and B1-WC are very similar, and the correction mainly results in a rigid shift of conduction and valence bands corresponding to a significant increase in the band gap. Using the B1-WC functional, the $E_{\mathrm{G}}$ values are 2.53, 

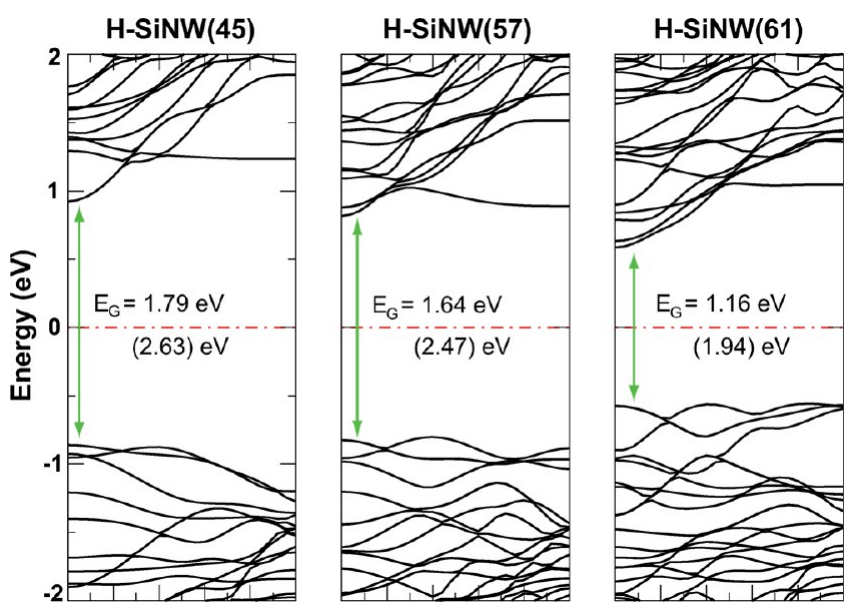

Figure 2. Band structures of hydrogen-saturated silicon nanowires along the [001] direction for $N=45,57$, and 61 . The electronic energy band structures and the gaps $\left(E_{\mathrm{G}}\right)$ are calculated using GGA. The values given in parentheses are obtained using B1-WC calculations.

2.38, and $1.81 \mathrm{eV}$ for $N=45,57$, and 61, respectively, as shown in Figure 2. For comparison, we also calculated $E_{\mathrm{G}}$ values with the HSE hybrid functional ${ }^{38,51,52}$ that provides values (2.63, 2.47, and $1.94 \mathrm{eV}$ for $N=45,57$, and 61, respectively) very close to the B1-WC results. Both hybrid functionals yield values in good agreement with the experimentally available data ${ }^{8}$ and theoretical GW corrected results ${ }^{15}$ for $\mathrm{H}-\mathrm{SiNW}$ sithin the same diameter range. ${ }^{43}$

\section{INTERSTITIAL TRANSITION-METAL DOPING}

Previously, we showed that TM atoms can strongly bind to $\mathrm{H}$ $\operatorname{SiNW}(N)$ surfaces without deforming the nanowire structure. $^{20,21}$ Because the doping occurs at high temperatures, there is a strong possibility that TM atom can also diffuse to interior regions. Accordingly, we extend our previous analysis and consider various possible interstitial sites for six TM atoms $(\mathrm{Ti}$, $\mathrm{V}, \mathrm{Cr}, \mathrm{Mn}, \mathrm{Fe}$, and $\mathrm{Co}$ ) and analyze energetics, structural, electronic, and magnetic properties of TM-doped H-SiNWs, labeled as $\mathrm{H}-\mathrm{SiNW}(N)+\mathrm{TM}(s)$, where $\mathrm{TM}$ is the type of transition-metal atom and $s$ is the interstitial doping site, as shown in Figure 3. As discussed in the Methodology section, we consider a supercell corresponding to a fix dopant

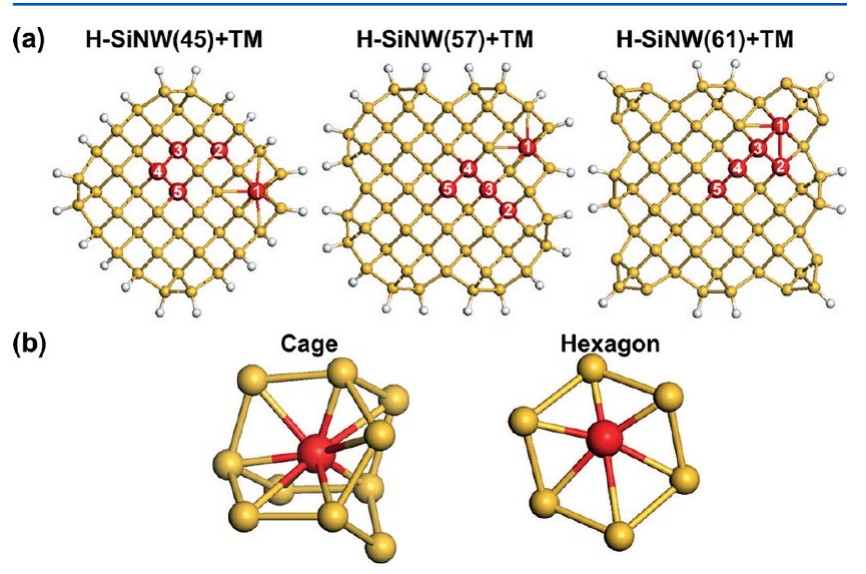

Figure 3. (a) Possible interstitial adsorption sites of transition-metal (red spheres) atoms labeled $1-5$ in $\mathrm{H}-\mathrm{SiNW}(N)$ for $N=45,57$, and 61 and (b) two alternative doping geometries. concentration associated to a distance between consecutive impurities of $5.4 \AA$. The obtained results, namely, bond distances $\left(d_{\mathrm{Si}-\mathrm{TM}}\right)$, binding energies $\left(E_{\mathrm{b}}\right)$, magnetic moments $(\mu)$, and the differences $(\Delta E)$ between the total energies of spin-unpolarized (su) and spin-polarized (sp) states are summarized in Tables 1 and 2.

Structure and Energetics. As a first step, we consider different positions from subsurface to core regions at which TM atoms can settle. The projection of these sites are shown in Figure 3. This corresponds to one impurity atom for six layers of $\mathrm{H}-\mathrm{SiNW}$ (Figure 1). For each site, there are two possible geometries for TM doping: the center of a cage, where TM atom is surrounded by ten silicon atoms, and the center of hexagon, where TM atom is in the middle of six silicon atoms, forming an almost planar geometry, as shown in Figure 3. For all cases, the cage geometry yields stronger $E_{\mathrm{b}}$ due to an increase in coordination number of TM atom. For most of the cases, the hexagon geometry is not stable, and the TM atom moves to the center of the silicon cage. Accordingly, we label the results corresponding to hexagon geometry with “*” and otherwise mention that the discussions are for TM atoms that are bound in cage geometry.

TM atoms can settle inside $\mathrm{H}-\mathrm{SiNW}(N)$ without deforming the wire structure for core sites $(s=3-5)$, and no structural difference is observed for different facets (Figure 3). Edge effects become more important for subsurface sites. Whereas no significant structural modification is observed for the $\mathrm{H}$ SiNW(61)+TM system, the $\mathrm{Si}-\mathrm{Si}$ bond is broken upon TM impurity for $\mathrm{H}-\mathrm{SiNW}(45)+\mathrm{TM}(1)$ and $\mathrm{H}-\mathrm{SiNW}(57)+\mathrm{TM}(1)$, as shown in Figure 3 . The minimum and maximum $d_{\mathrm{Si}-\mathrm{TM}}$ ranges between 2.2 and $2.9 \AA$ (generally 2.4 to $2.8 \AA$ ) are summarized in Tables 1 and 2 for subsurface and core sites, respectively.

On the one hand, the comparison of $E_{\mathrm{T}}$ indicates that the bond-breaking at site (1) for H-SiNW(45) and H-SiNW(57) results in an increase in $E_{\mathrm{T}}$ and makes it less favorable than other possible subsurface sites $(s=2)$ where there is no deformation. On the other hand, for the case of $\mathrm{H}-\mathrm{SiNW}(61)$, site (1), which is inside a sharp corner (edge), is the lowest energy configuration. For core sites, $E_{\mathrm{T}}$ values slightly vary while moving from subsurface to the center $(3 \rightarrow 5)$, and the influence of surface becomes less significant.

We calculate the binding energy $\left(E_{\mathrm{b}}\right)$ of $\mathrm{TM}$ atoms for different sites by using the expression

$$
\begin{aligned}
E_{\mathrm{b}}= & \mathrm{E}_{\mathrm{T}}[\mathrm{H}-\mathrm{SiNW}(N)]+\mathrm{E}_{\mathrm{T}}[\mathrm{TM}]-\mathrm{E}_{\mathrm{T}}[\mathrm{H}-\operatorname{SiNW}(N) \\
& +\mathrm{TM}]
\end{aligned}
$$

in terms of the total energy of optimized $\mathrm{H}-\mathrm{SiNW}(\mathrm{N})$ and $\mathrm{H}$ $\operatorname{SiNW}(N)+\mathrm{TM}$ and the total energy of the string of TM atoms having the same lattice parameter as $\mathrm{H}-\operatorname{SiNW}(N)+\mathrm{TM}$, all calculated in the same supercell. Interestingly, the variation of $E_{\mathrm{b}}$ with the type of TM atoms follows the Friedel model ${ }^{53}$ for both different sites and facets (Figure 4). ${ }^{54,55}$ Accordingly, the lowest $E_{\mathrm{b}}$ is obtained for $\mathrm{Cr}$ and $\mathrm{Mn}$, and the highest $E_{\mathrm{b}}$ is obtained for $\mathrm{Co}$ and $\mathrm{Ti}$, depending on the number of filled $\mathrm{d}$ states. When different facets are compared, $E_{\mathrm{b}}$ for $N=61$ is higher than $N=57$ and 45 for subsurfaces sites, and there is no significant variation noticed for core sites. When doping sites are examined, interestingly, the highest $E_{\mathrm{b}}$ is obtained at sites (5), (2), and (1) for $\mathrm{H}-\operatorname{SiNW}(45), \mathrm{H}-\operatorname{SiNW}(57)$, and $\mathrm{H}-$ SiNW(61), respectively. The results point out that the 
Table 1. Bond Distances, Binding Energy, Magnetic Moment Per Cell, and Total Energy Difference of Polarized and Unpolarized States for Subsurface $(s=1$ and 2$)$ Sites

\begin{tabular}{|c|c|c|c|c|c|c|c|c|c|c|c|c|c|}
\hline & \multirow[b]{2}{*}{$s$} & \multicolumn{4}{|c|}{$N=45$} & \multicolumn{4}{|c|}{$N=57$} & \multicolumn{4}{|c|}{$N=61$} \\
\hline & & $d_{\mathrm{Si}-\mathrm{TM}}(\AA)$ & $E_{\mathrm{b}}(\mathrm{eV})$ & $\mu\left(\mu_{\mathrm{b}}\right)$ & $\Delta E(\mathrm{eV})$ & $d_{\mathrm{Si}-\mathrm{TM}}(\AA)$ & $E_{\mathrm{b}}(\mathrm{eV})$ & $\mu\left(\mu_{\mathrm{b}}\right)$ & $\Delta E(\mathrm{eV})$ & $d_{\mathrm{Si}-\mathrm{TM}}(\AA)$ & $E_{\mathrm{b}}(\mathrm{eV})$ & $\mu\left(\mu_{\mathrm{b}}\right)$ & $\Delta E(\mathrm{eV})$ \\
\hline \multirow[t]{2}{*}{$\mathrm{Ti}$} & 1 & $2.38-2.85$ & 3.42 & 0.00 & 0.00 & $2.38-2.86$ & 3.59 & 0.00 & 0.00 & $2.47-2.98$ & 4.34 & 0.00 & 0.00 \\
\hline & 2 & $2.43-2.84$ & 3.59 & 0.00 & 0.00 & $2.45-2.80$ & 3.92 & 0.00 & 0.00 & $2.42-2.85$ & 4.35 & 0.00 & 0.00 \\
\hline \multirow[t]{2}{*}{ V } & 1 & $2.34-2.77$ & 2.96 & 1.01 & 0.02 & $2.34-2.78$ & 3.07 & 0.83 & 0.02 & $2.45-2.83$ & 3.82 & 2.97 & 0.02 \\
\hline & 2 & $2.42-2.78$ & 3.09 & 1.21 & 0.02 & $2.39-2.75$ & 3.38 & 1.01 & 0.01 & $2.39-2.78$ & 3.39 & 1.39 & 0.02 \\
\hline \multirow[t]{2}{*}{$\mathrm{Cr}$} & 1 & $2.29-2.73$ & 1.42 & 3.75 & 0.09 & $2.31-2.97$ & 1.72 & 3.17 & 0.21 & $2.42-2.80$ & 2.46 & 2.94 & 0.46 \\
\hline & 2 & $2.45-2.75$ & 1.54 & 3.72 & 0.20 & $2.43-2.73$ & 1.87 & 3.51 & 0.26 & $2.41-2.81$ & 1.89 & 3.46 & 0.27 \\
\hline \multirow[t]{2}{*}{$\mathrm{Mn}$} & 1 & $2.27-2.77$ & 1.75 & 2.53 & 0.05 & $2.27-2.86$ & 1.89 & 2.44 & 0.10 & $2.40-2.77$ & 2.66 & 2.39 & 0.42 \\
\hline & 2 & $2.42-2.77$ & 1.93 & 3.00 & 0.35 & $2.41-2.75$ & 2.21 & 3.00 & 0.42 & $2.39-2.81$ & 2.22 & 3.00 & 0.39 \\
\hline \multirow[t]{2}{*}{$\mathrm{Fe}$} & 1 & $2.23-2.74$ & 3.18 & 1.55 & 0.19 & $2.22-2.85$ & 3.28 & 1.46 & 0.19 & $2.35-2.74$ & 3.83 & 1.39 & 0.35 \\
\hline & 2 & $2.39-2.73$ & 3.26 & 2.00 & 0.35 & $2.37-2.73$ & 3.45 & 2.00 & 0.31 & $2.36-2.81$ & 3.44 & 2.00 & 0.28 \\
\hline \multirow[t]{2}{*}{ Co } & 1 & $2.17-3.01$ & 3.94 & 0.00 & 0.00 & $2.19-2.97$ & 4.16 & 0.00 & 0.00 & $2.30-2.96$ & 4.76 & 0.00 & 0.00 \\
\hline & 2 & $2.39-2.70$ & 3.80 & 1.00 & 0.08 & $2.37-2.77$ & 3.99 & 1.00 & 0.03 & $2.37-2.89$ & 4.02 & 1.00 & 0.02 \\
\hline
\end{tabular}

Table 2. Bond Distances, Binding Energy, Magnetic Moment Per Cell, and Total Energy Difference of Polarized and Unpolarized States for Core $\left(s=3,4\right.$, and 5) Sites ${ }^{a}$

\begin{tabular}{|c|c|c|c|c|c|c|c|c|c|c|c|c|c|}
\hline & & & $N=$ & & & & $N=$ & & & & $N=$ & & \\
\hline & $s$ & $d_{\mathrm{Si}-\mathrm{TM}}(\AA)$ & $E_{\mathrm{b}}(\mathrm{eV})$ & $\mu\left(\mu_{b}\right)$ & $\Delta E(\mathrm{eV})$ & $d_{\mathrm{Si}-\mathrm{TM}}(\AA)$ & $E_{\mathrm{b}}(\mathrm{eV})$ & $\mu\left(\mu_{\mathrm{b}}\right)$ & $\Delta E(\mathrm{eV})$ & $d_{\mathrm{Si}-\mathrm{TM}}(\AA)$ & $E_{\mathrm{b}}(\mathrm{eV})$ & $\mu\left(\mu_{\mathrm{b}}\right)$ & $\Delta E(\mathrm{eV})$ \\
\hline $\mathrm{Ti}$ & 3 & $2.45-2.78$ & 3.65 & 0.00 & 0.00 & $2.44-2.84$ & 3.86 & 0.00 & 0.00 & $2.42-2.85$ & 3.95 & 0.00 & 0.00 \\
\hline & 4 & $2.45-2.78$ & 3.65 & 0.00 & 0.00 & $2.47-2.80$ & 3.75 & 0.00 & 0.00 & $2.45-2.79$ & 3.72 & 0.00 & 0.00 \\
\hline & 5 & $2.45-2.83$ & 3.72 & 0.00 & 0.00 & $2.48-2.73$ & 3.57 & 0.00 & 0.00 & $2.47-2.79$ & 3.78 & 0.00 & 0.00 \\
\hline $\mathrm{V}$ & 3 & $2.44-2.73$ & 3.20 & 2.52 & 0.08 & $2.42-2.80$ & 3.33 & 1.36 & 0.03 & $2.39-2.78$ & 3.39 & 1.40 & 0.02 \\
\hline & 4 & $2.44-2.73$ & 3.20 & 2.52 & 0.08 & $2.43-2.76$ & 3.24 & 1.67 & 0.03 & $2.42-2.77$ & 3.28 & 1.32 & 0.09 \\
\hline & 5 & $2.41-2.79$ & 3.19 & 1.41 & 0.02 & $2.46-2.79$ & 3.19 & 2.42 & 0.12 & $2.40-2.80$ & 3.21 & 2.05 & 0.03 \\
\hline $\mathrm{Cr}$ & 3 & $2.45-2.75$ & 1.78 & 4.00 & 0.40 & $2.42-2.79$ & 1.78 & 3.61 & 0.25 & $2.41-2.81$ & 1.89 & 3.46 & 0.27 \\
\hline & 4 & $2.45-2.75$ & 1.78 & 4.00 & 0.40 & $2.45-2.76$ & 1.79 & 3.82 & 0.34 & $* 2.44-2.80$ & $* 1.83$ & $* 4.00$ & $* 0.34$ \\
\hline & 5 & $2.43-2.78$ & 1.73 & 4.00 & 0.33 & $2.47-2.70$ & 1.77 & 4.00 & 0.38 & $2.32-2.46$ & 1.91 & 4.00 & 0.38 \\
\hline $\mathrm{Mn}$ & 3 & $* 2.37-2.39$ & $* 1.45$ & $* 3.00$ & $* 0.62$ & $2.40-2.79$ & 2.18 & 3.00 & 0.47 & $2.39-2.81$ & 2.22 & 3.00 & 0.39 \\
\hline & 4 & $* 2.32-2.38$ & $* 1.54$ & $* 3.00$ & $* 0.60$ & $2.42-2.77$ & 2.14 & 3.00 & 0.45 & $* 2.33-2.37$ & $* 1.49$ & $* 3.00$ & $* 0.35$ \\
\hline & 5 & $2.40-2.79$ & 2.09 & 3.00 & 0.52 & $2.44-2.69$ & 2.06 & 3.00 & 0.36 & $* 2.31-2.38$ & $* 1.61$ & $* 3.00$ & $* 0.32$ \\
\hline $\mathrm{Fe}$ & 3 & $* 2.26-2.38$ & $* 2.74$ & $* 2.00$ & $* 0.44$ & $2.38-2.77$ & 3.41 & 2.00 & 0.38 & $2.36-2.81$ & 3.44 & 2.00 & 0.28 \\
\hline & 4 & $* 2.26-2.39$ & $*_{2.84}$ & $*_{2.00}$ & $*_{0.40}$ & $2.39-2.76$ & 3.38 & 2.00 & 0.34 & $* 2.27-2.37$ & $*_{2.81}$ & $* 2.00$ & $* 0.41$ \\
\hline & 5 & $2.38-2.78$ & 3.34 & 2.00 & 0.45 & $2.42-2.69$ & 3.35 & 2.00 & 0.27 & $* 2.26-2.39$ & $* 2.89$ & $* 2.00$ & $* 0.29$ \\
\hline Co & 3 & $* 2.28-2.34$ & $* 3.60$ & $* 1.00$ & $* 0.06$ & $2.36-2.79$ & 3.92 & 1.00 & 0.07 & $2.37-2.89$ & 4.02 & 1.00 & 0.03 \\
\hline & 4 & $* 2.28-2.33$ & $* 3.68$ & $* 1.00$ & $* 0.02$ & $2.37-2.82$ & 3.94 & 1.00 & 0.06 & $2.37-2.89$ & 3.64 & 1.00 & 0.04 \\
\hline & 5 & $2.37-2.75$ & 3.85 & 1.00 & 0.08 & $2.42-2.70$ & 3.95 & 1.00 & 0.05 & $* 2.28-2.34$ & $* 3.83$ & $* 1.00$ & $* 0.03$ \\
\hline
\end{tabular}

${ }^{a}$ Results indicated by “*” correspond to hexagonal symmetry. (See the text.)
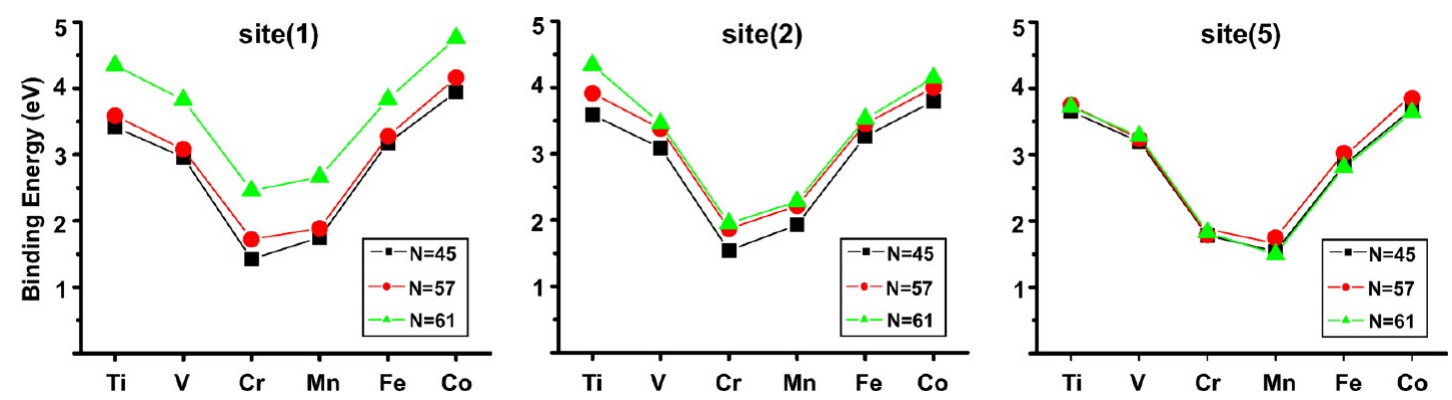

Figure 4. Binding energy trend with respect to type of transition-metal atoms ( $\mathrm{Ti}, \mathrm{V}, \mathrm{Cr}, \mathrm{Mn}, \mathrm{Fe}$, and $\mathrm{Co}$ ) in $\mathrm{H}-\mathrm{SiNW}(\mathrm{N})$ for $N=45,57$, and 61 for the sites 1,2 (subsurface), and 5 (core).

structural properties as well as the energetics depend on both the type of TM and the cross section due to surface effects.

Magnetic Properties. The total energies $\left(E_{\mathrm{T}}\right)$ for the considered systems are obtained from both $\mathrm{sp}$ and su states, and the energy difference $\left(\Delta E=E_{\mathrm{T}}^{\mathrm{su}}-E_{\mathrm{T}}^{\mathrm{sp}}\right)$ is used to determine the lowest energy configuration. According to this definition, the sp state is the lowest energy configuration when $\Delta E>0$. As shown in Tables 1 and 2, except for $\mathrm{Ti}$, the lowest energy configuration is obtained for sp state. Additionally, we also compared the $E_{\mathrm{T}}$ values in ferromagnetic and antiferromagnetic states and confirmed that the ground state is ferromagnetic, except $\mathrm{Ti}$, which yields paramagnetic ground state. The 

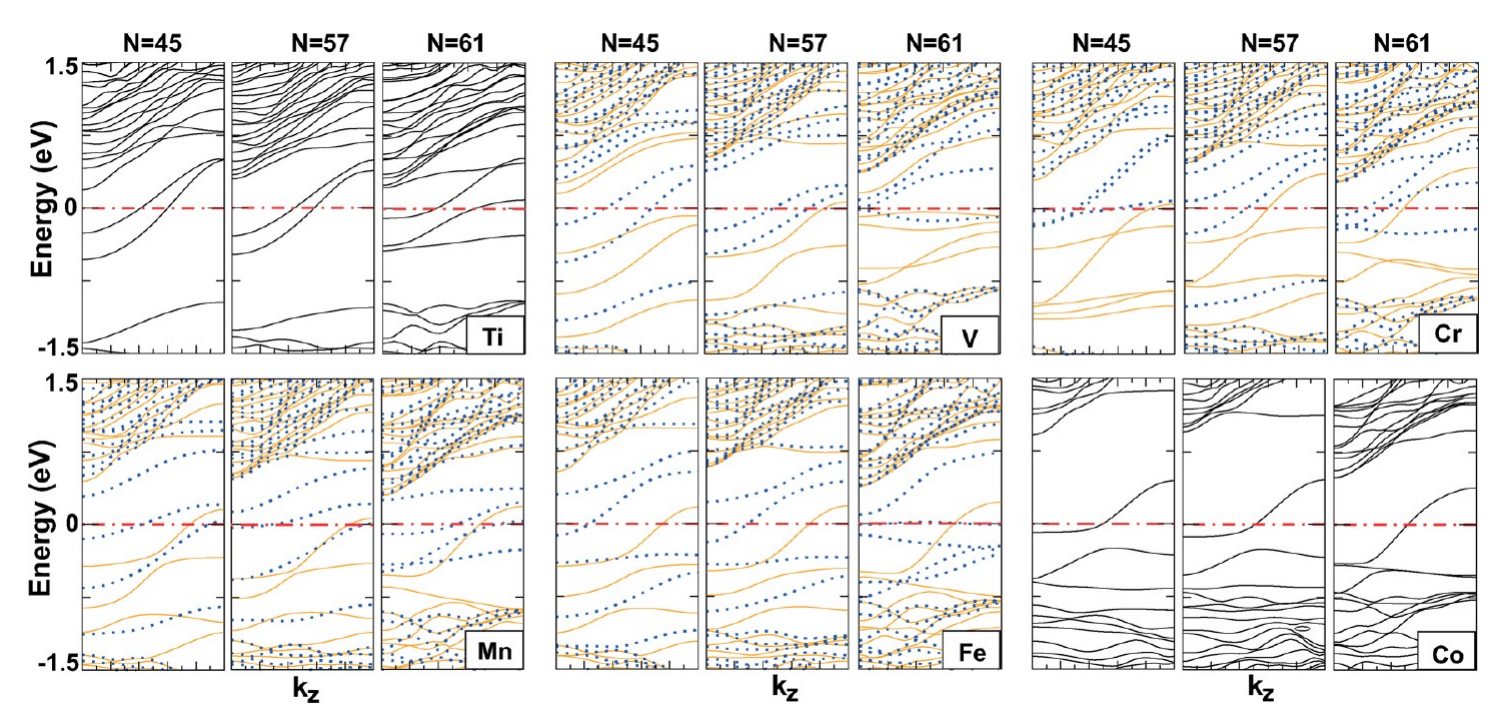

Figure 5. Electronic band structures of $\mathrm{H}-\mathrm{SiNW}(N)+\mathrm{TM}$ at site (1) for $N=45,57$, and 61 and $\mathrm{TM}=\mathrm{Ti}, \mathrm{V}, \mathrm{Cr}, \mathrm{Mn}, \mathrm{Fe}$, and Co. Solid and dotted lines indicate spin up and down states, respectively.
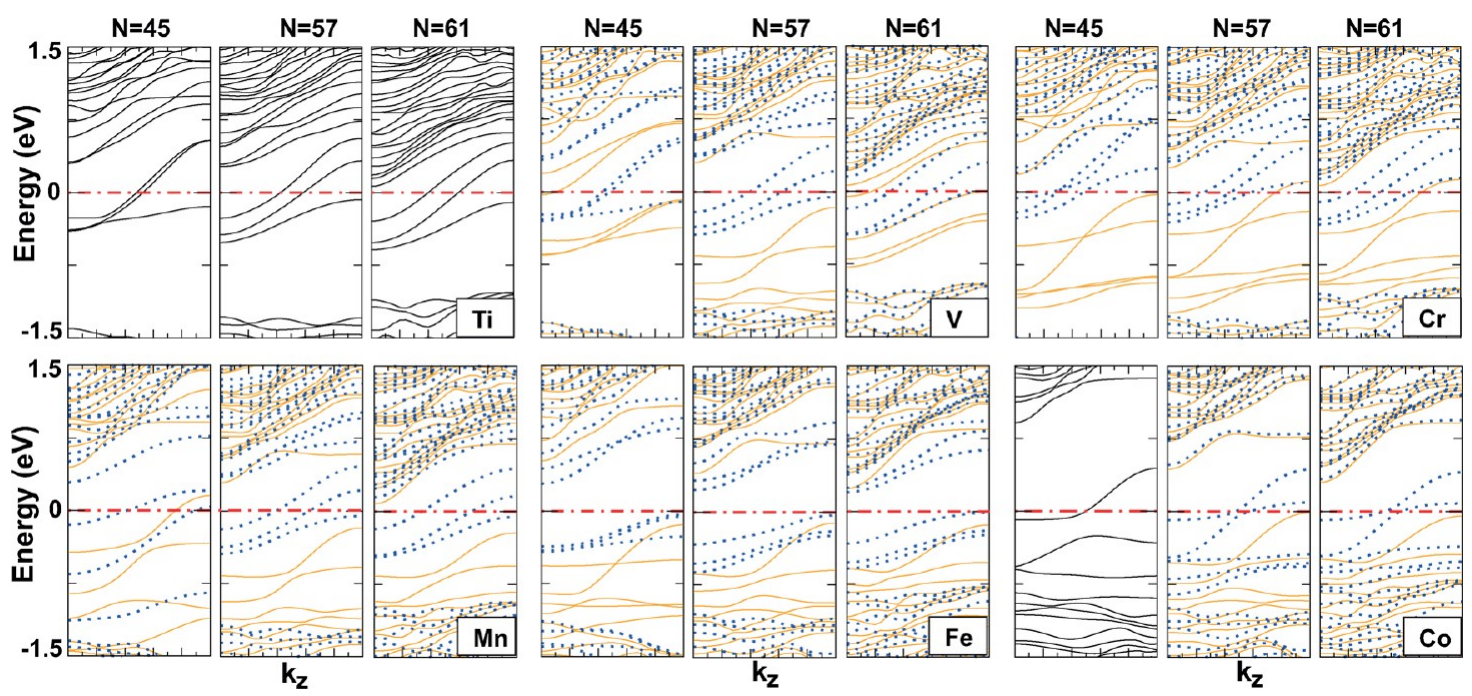

Figure 6. Electronic band structures of $\mathrm{H}-\mathrm{SiNW}(N)+\mathrm{TM}$ at site (2) for $N=45,57$, and 61 and $\mathrm{TM}=\mathrm{Ti}, \mathrm{V}, \mathrm{Cr}, \mathrm{Mn}, \mathrm{Fe}$, and Co. Solid and dotted lines indicate spin up and down states, respectively.
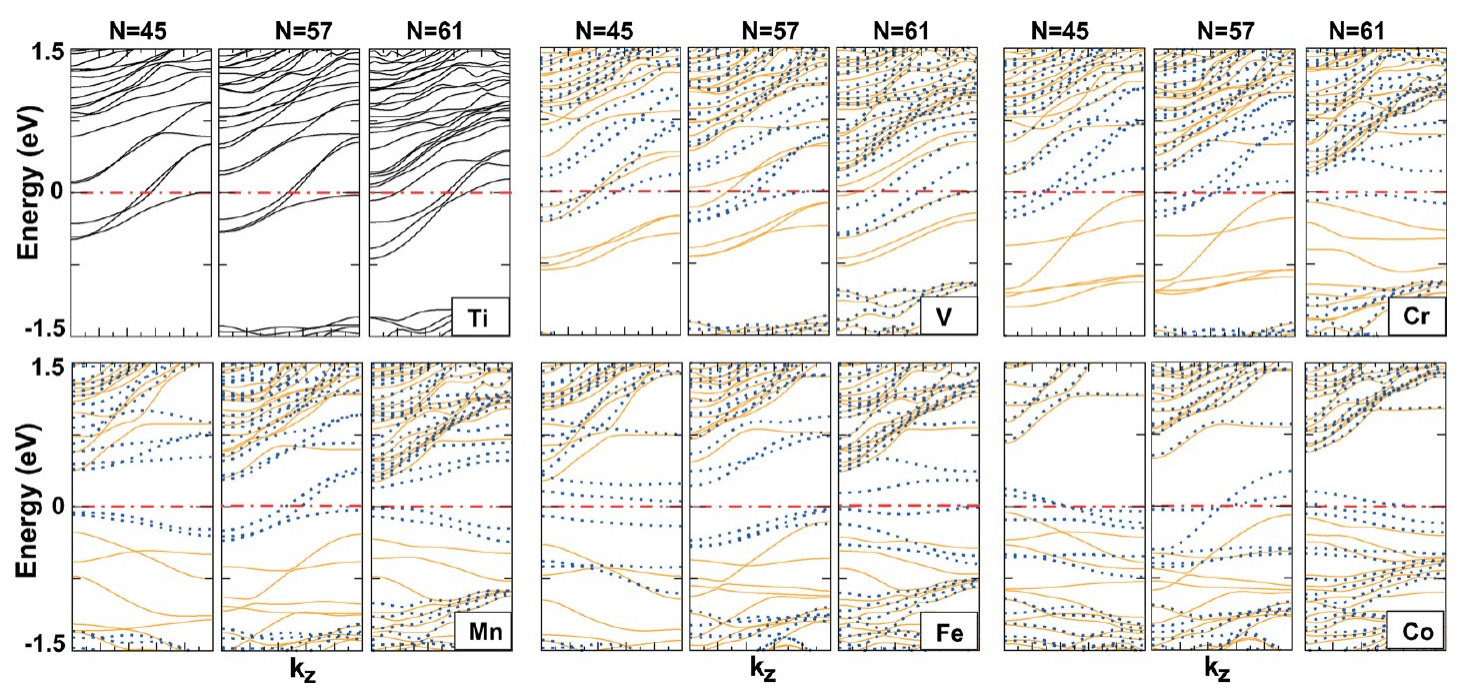

Figure 7. Electronic band structures of $\mathrm{H}-\mathrm{SiNW}(N)+\mathrm{TM}$ at site (4) for $N=45,57$, and 61 and $\mathrm{TM}=\mathrm{Ti}, \mathrm{V}, \mathrm{Cr}, \mathrm{Mn}, \mathrm{Fe}$, and Co. Solid and dotted lines indicate spin up and down states, respectively. 

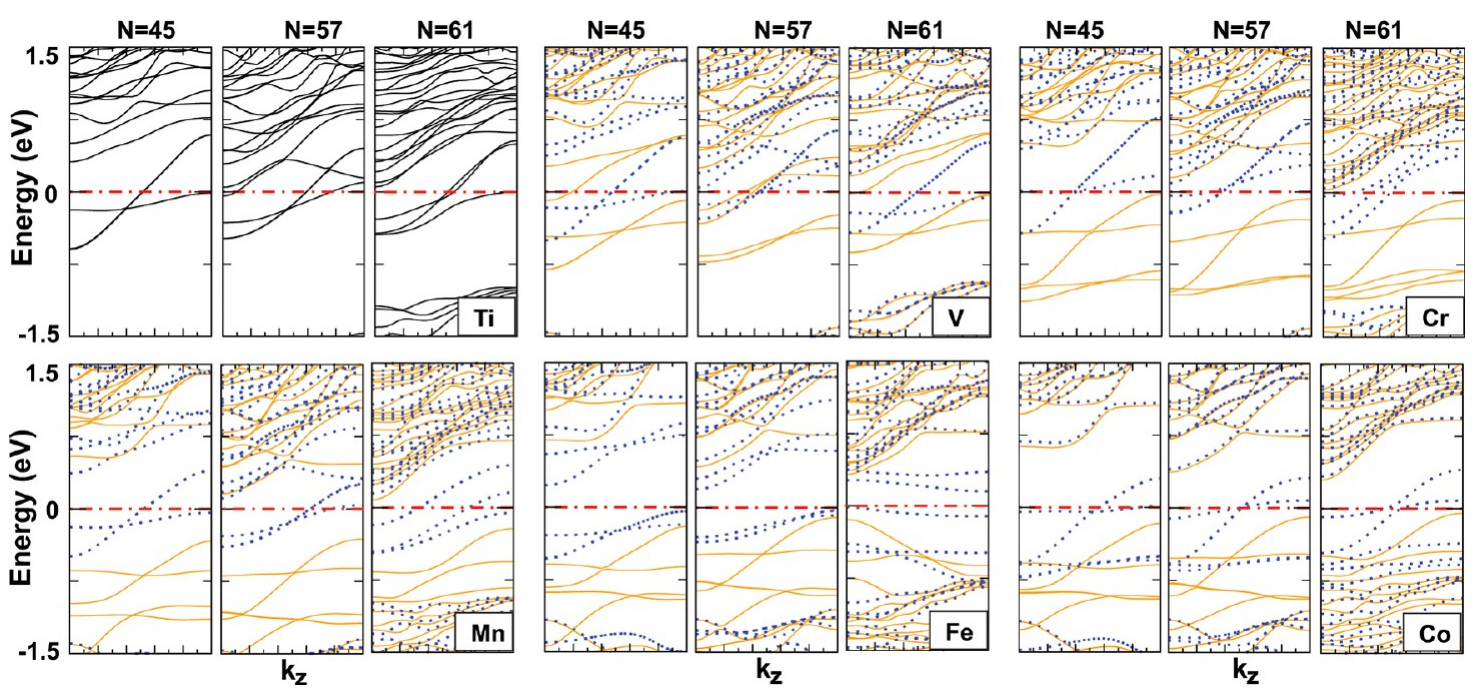

Figure 8. Electronic band structures of $\mathrm{H}-\mathrm{SiNW}(N)+\mathrm{TM}$ at site (5) for $N=45,57$, and 61 and $\mathrm{TM}=\mathrm{Ti}, \mathrm{V}, \mathrm{Cr}, \mathrm{Mn}, \mathrm{Fe}$, and Co. Solid and dotted lines indicate spin up and down states, respectively.

magnetic moments, $\mu$, vary depending on both the type of TM and the doping site, as listed in Tables 1 and 2.

Using the energy difference between ferromagnetic and antiferromagnetic states, we can roughly estimate the Curie temperature $\left(T_{\mathrm{C}}\right)$ in the mean field approximation. Accordingly, we considered $\mathrm{H}-\mathrm{SiNW}(45)+\mathrm{TM}$ as the prototype and calculated $T_{\mathrm{C}}$ to be $\sim 600,900$, and $400 \mathrm{~K}$, for $\mathrm{Cr}, \mathrm{Mn}$, and $\mathrm{Fe}$, respectively, which suggests that the ferromagnetic state should be stable over room temperature. In contrast, $T_{\mathrm{C}}$ for $\mathrm{V}$ and $\mathrm{Co}$ is $\sim 50 \mathrm{~K}$, which shows that they can have ferromagnetic ground state only at very low temperatures. Finally, Ti yields paramagnetic ground state even at $0 \mathrm{~K}$. When the trend of $T_{\mathrm{C}}$ is compared with the unpaired d electrons ( $\left.d^{\text {un }}\right)$ of TM dopants, a strong relation can be noticed. When $d^{\text {un }}=2(\mathrm{Ti})$, $T_{\mathrm{C}}=0$ (in other words paramagnetic); $d^{\text {un }}=3(\mathrm{~V}, \mathrm{Co}), T_{\mathrm{C}} \simeq$ 50; $d^{\mathrm{un}}=4(\mathrm{Fe}), T_{\mathrm{C}} \simeq 400$; and $d^{u n}=5(\mathrm{Cr}$ and $\mathrm{Mn}), T_{\mathrm{C}}>600$. Accordingly, we can conclude that the stability of ferromagnetic state is mainly determined by the number of unpaired $d$ states.

Electronic Structure. Our calculations reveal that electronic structures are strongly affected by the type of TM atoms, doping site, and also cross section of $\mathrm{H}-\operatorname{SiNW}(N)$. The resulting band structures for subsurface $(s=1,2)$ and core sites $(s=4,5)$ are shown in Figures 5-8. Depending on the configuration, the ground state can be paramagnetic or ferromagnetic, and the electronic structure can be metal, semiconductor, semimetal, or half-metal. ${ }^{20,21}$

If we analyze the systems one-by-one, then the $\mathrm{H}$ $\operatorname{SiNW}(N)+\mathrm{Ti}$ configuration is a paramagnetic metal for all considered systems. Interestingly, for $\mathrm{V}$ at site (1), the nanowires become half-metal, metal, and semimetal for $N=$ 45,57 , and 61 , respectively, and this indicates the effect of cross section on the electronic structure. The half-metallic state is also noticed for $N=57$ at site (2), and for all other doping sites $(s=3-5)$, the system is ferromagnetic metal with varying magnetic moment. $\mathrm{Cr}$ and $\mathrm{Mn}$ behave very similarly. The nanowires are, in general, ferromagnetic metal for subsurface sites, and they become half-metallic for core regions. Exceptionally, $\mathrm{H}-\mathrm{SiNW}(61)+\mathrm{Cr}(4)$ and $\mathrm{H}-\mathrm{SiNW}(61)+\mathrm{Mn}(4,5)$ are ferromagnetic semiconductors because $\mathrm{TM}$ is bound to hexagonal site instead of cage configuration (Figure 3). For $\mathrm{Fe}$, all $\mathrm{H}-\mathrm{SiNW}$ are ferromagnetic semimetal at site (1), but they become ferromagnetic semiconductor for all other sites including hexagonal doping site. Finally, for Co, all H-SiNWs are paramagnetic metal at site (1) and become half-metal or ferromagnetic semiconductor for core regions.

Combining the results from the previous sections we can conclude that: (i) The energetics of binding are almost identical at core sites for different types H-SiNWs, but the variation is noticed for subsurface sites due to surface effects. (ii) The trend of $E_{\mathrm{b}}$ follows Friedel model ${ }^{53}$ independent of $\mathrm{H}$-SiNW type and binding site. (iii) The electronic ground state of H-SiNW$(N)+$ TM is mainly determined by the type of (or the d-electron configuration of) TM dopants but can also differ for the same TM depending on the doping site and geometry. (iv) TM doping generally induces metallization (either paramagnetic or ferromagnetic) except for the case of Fe. H-SiNW $(N)$ s still remain semiconducting after $\mathrm{Fe}$ doping with varying electronic band gap. The semimetallic behavior of $\mathrm{H}-\mathrm{SiNW}(N)+\mathrm{Fe}(1)$ is an exceptional case and will be further discussed in the next section. (v) $\mathrm{H}-\mathrm{SiNW}(\mathrm{N})+(\mathrm{Cr}, \mathrm{Mn})$ systems are, in general, ferromagnetic metal for subsurface sites, and they start to possess novel half-metallic behavior for core sites. (vi) However, $\mathrm{H}-\mathrm{SiNW}(\mathrm{N})+\mathrm{V}$ and $\mathrm{H}-\mathrm{SiNW}(N)+\mathrm{Co}$ also have half-metallic ground state for specific cases; they are ferromagnetic only at very low temperatures, which makes them practically paramagnetic metals. Accordingly, stable halfmetallic ground state is unique to $\mathrm{Cr}$ and $\mathrm{Mn}$, where all of the $\mathrm{d}$-electrons are unpaired. (vii) When $\mathrm{Mn}$ and $\mathrm{Cr}$ are settled in the center of silicon hexagonal plane, $d_{\mathrm{Si}-\mathrm{TM}}$ becomes equal for all six nearest silicon atoms, and it leads to different hybridization of $\mathrm{p}$ (that belongs to $\mathrm{H}$-SiNW) and $\mathrm{d}$ orbitals (that belong to TM), which makes the systems semiconductor instead of half-metal. However this configuration is energetically less favorable when compared with cage geometry but can be stable.

Evolution with Dopant Concentration. All previous calculations have been performed at a fixed doping concentration corresponding to a distance between impurities of $5.4 \AA$. Even if our aim is not specifically to determine shallow impurity levels, it is interesting to clarify the range of interaction between $\mathrm{TM}$ atoms and the way the properties evolve with the distance between impurity atoms. To that end, we now consider the prototype $\mathrm{H}-\mathrm{SiNW}(45)+\mathrm{Cr}(5)$ case and increase the supercell size along [001] direction from $n=1$ up to $n=5$ unit cell of 
nanowires, so increasing the distance between impurity up to $27 \AA$ and decreasing the doping concentration to $0.3 \%$.

The results of our analysis are summarized in Table 3 . The bond length and magnetic moments are essentially not affected

Table 3. Bond Lengths, Binding Energy, Magnetic Moment Per Cell, Total Energy Difference of Polarized, and Unpolarized States for 1 to 5 Unit Cells of $\mathrm{H}-\mathrm{SiNW}(45)+\mathrm{Cr}$ at Site (5)

\begin{tabular}{cccc} 
size & $d_{\mathrm{Si}-\mathrm{TM}}(\AA)$ & $E_{\mathrm{b}}(\mathrm{eV})$ & $\mu\left(\mu_{\mathrm{b}}\right)$ \\
1 & $2.43-2.78$ & 1.726 & 4.00 \\
2 & $2.43-2.78$ & 1.810 & 4.00 \\
3 & $2.43-2.78$ & 1.813 & 4.00 \\
4 & $2.43-2.78$ & 1.809 & 4.00 \\
5 & $2.43-2.78$ & 1.811 & 4.00 \\
\hline
\end{tabular}

by the supercell size. As expected, $E_{\mathrm{b}}$ slightly increases with $n$ owing to the reduction in TM-TM interaction (which enhances TM-SiNW interaction) but is already converged for $n=2$. The same kind of evolution has been obtained for other impurities. ${ }^{56}$

Similar trends are observed in the band structures shown in Figure 9. Dispersionless impurity levels rapidly emerge and can

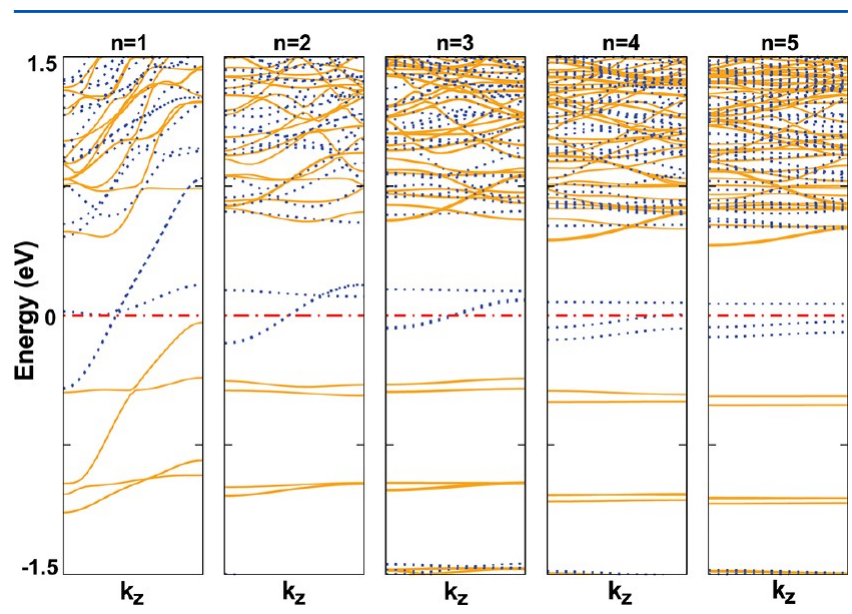

Figure 9. Electronic band structures of $\mathrm{H}-\mathrm{SiNW}(61)+\mathrm{Cr}$ site (5) for unit cell size $1-5$. Solid and dotted lines indicate spin up and down states, respectively.

be observed starting from $n=4$ or 5 , corresponding to distances on the order of 20-25 A between $\mathrm{Cr}$ atoms. Both valence and conduction band edges belong to minority spin states, indicating half-metallic nature of the system.

\section{SUBSTITUTIONAL TRANSITION-METAL DOPING}

Even though we mainly focus on interstitial impurities, we also consider substitutional doping for all types of impurities at one subsurface and one core site (site 1 and 5) of H-SiNW(61). The results are summarized in Tables 4 and 5 . Our results indicate that TM atoms can also substitute $\mathrm{Si}$ atoms without deforming $\mathrm{H}$-SiNW. For all impurities, the subsurface substitution is more favorable energetically than the core one. $d_{\mathrm{Si}-\mathrm{TM}}$ changes between 2.1 and $2.8 \AA$, slightly shorter than the interstitial counterpart. Whereas TM atoms at the subsurface site have six- to seven-fold coordinations, they have four-fold coordinations at the core site. Instead of $E_{\mathrm{b}}$ we defined
Table 4. Bond Distances, Formation Energy, Magnetic Moment Per Cell, and Total Energy Difference of Polarized and Unpolarized States of H-SiNW(61)+TM System for Subsurface Substitution

\begin{tabular}{lcccc} 
& $d_{\mathrm{Si}-\mathrm{TM}}(\AA)$ & $E_{\mathrm{f}}(\mathrm{eV})$ & $\mu\left(\mu_{\mathrm{b}}\right)$ & $\Delta E(\mathrm{eV})$ \\
$\mathrm{Ti}$ & $2.49-2.75$ & -2.01 & 0.00 & 0.00 \\
$\mathrm{~V}$ & $2.28-2.45$ & -1.16 & 0.00 & 0.00 \\
$\mathrm{Cr}$ & $2.36-2.76$ & -2.65 & 2.00 & 0.40 \\
$\mathrm{Mn}$ & $2.21-2.70$ & -1.74 & 1.71 & 0.15 \\
$\mathrm{Fe}$ & $2.14-2.64$ & -1.58 & 0.00 & 0.00 \\
$\mathrm{Co}$ & $2.18-2.63$ & -1.30 & 0.00 & 0.00 \\
\hline
\end{tabular}

Table 5. Bond Distances, Formation Energy, Magnetic Moment Per Cell, Total Energy Difference of Polarized, and Unpolarized States of H-SiNW(61)+TM System for Core Substitution

\begin{tabular}{lcccc} 
& $d_{\mathrm{Si}-\mathrm{TM}}(\AA)$ & $E_{\mathrm{f}}(\mathrm{eV})$ & $\mu\left(\mu_{\mathrm{b}}\right)$ & $\Delta E(\mathrm{eV})$ \\
$\mathrm{Ti}$ & 2.51 & -2.93 & 0.00 & 0.00 \\
$\mathrm{~V}$ & 2.43 & -2.55 & 1.00 & 0.20 \\
$\mathrm{Cr}$ & 2.40 & -3.42 & 2.00 & 0.64 \\
$\mathrm{Mn}$ & 2.38 & -2.86 & 3.00 & 0.56 \\
$\mathrm{Fe}$ & 2.25 & -2.67 & 0.00 & 0.00 \\
$\mathrm{Co}$ & 2.24 & -2.37 & 0.47 & 0.02 \\
\hline
\end{tabular}

formation energy $\left(E_{\mathrm{f}}\right)$ for substitutional impurities, and it is expressed as

$$
\begin{aligned}
E_{\mathrm{f}}= & E_{\mathrm{T}}[\mathrm{H}-\mathrm{SiNW}(N)]+\mu[\mathrm{TM}]-\mathrm{E}_{\mathrm{T}}[\mathrm{H}-\operatorname{SiNW}(N) \\
& +\mathrm{TM}]-\mu[\mathrm{Si}]
\end{aligned}
$$

in terms of the total energy of optimized H-SiNW $(N)$ and $\mathrm{H}$ $\mathrm{SiNW}(N)+\mathrm{TM}$ and chemical potential of TM and $\mathrm{Si}$ atoms taken from the total energy of the string of TM atoms having the same lattice parameter as $\mathrm{H}-\operatorname{SiNW}(N)+\mathrm{TM}$ and total energy of $\mathrm{Si}$ atom in $\mathrm{SiNW}$. The negative value of $E_{\mathrm{f}}$ indicates the requirement of energy for such kind of substitution. It should also be noted that $\left|E_{\mathrm{f}}\right|$ is larger for the core case, indicating a larger energy requirement for core substitution. The variation magnetic properties follow a similar trend when compared with the interstitial doping except for Fe. Whereas Fe interstitial doping results in a ferromagnetic ground state, it yields a paramagnetic ground state for substitutional doping.

Electronic structure also varies depending on the type of impurity and site of substitution. The resulting band structures are shown in Figures 10 and 11. A similar electronic structure profile is obtained for interstitial and substitutional doping of $\mathrm{Fe}$ and Co cases. Wherease interstitial doping of $\mathrm{Ti}, \mathrm{Cr}$, and $\mathrm{Mn}$ yields metallic state, $\mathrm{H}-\mathrm{SiNW}(61)$ becomes semiconductor upon substitutional doping of the same atoms. The half-metallic state that formed for interstitial doping of $\mathrm{Cr}, \mathrm{Mn}$, and $\mathrm{Co}$ at the core site is not obtained for the substitutional case except for $\mathrm{V}$ doping.

\section{HYBRID FUNCTIONALS AND GGA+U}

It is well known that the usual approximations to DFT, such as LDA or GGA, lead to significant underestimation of the band gaps due to intrinsic failure in handling self-interactions. ${ }^{50} \mathrm{GW}$ corrections $^{57}$ appeared as a powerful tool to overcome this problem and were recently applied to silicon nanowires. ${ }^{15,58}$ Unfortunately, such GW calculations are computationally very costly, and it is difficult to apply this technique on large 

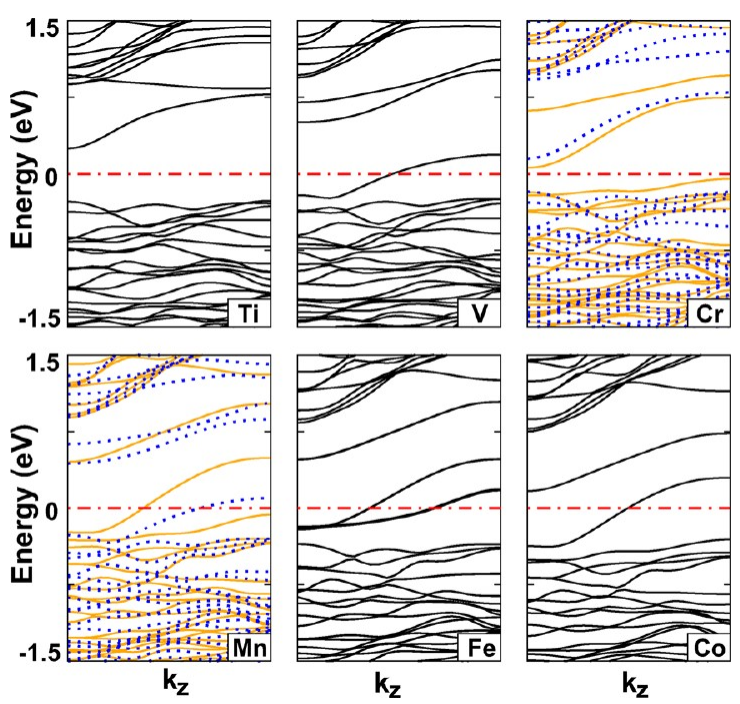

Figure 10. Electronic band structures of $\mathrm{H}-\mathrm{SiNW}(N)+\mathrm{TM}$ at site (1) for $N=45,57$, and 61 and $\mathrm{TM}=\mathrm{Ti}, \mathrm{V}, \mathrm{Cr}, \mathrm{Mn}, \mathrm{Fe}$, and $\mathrm{Co}$. Solid and dotted lines indicate spin up and down states, respectively.
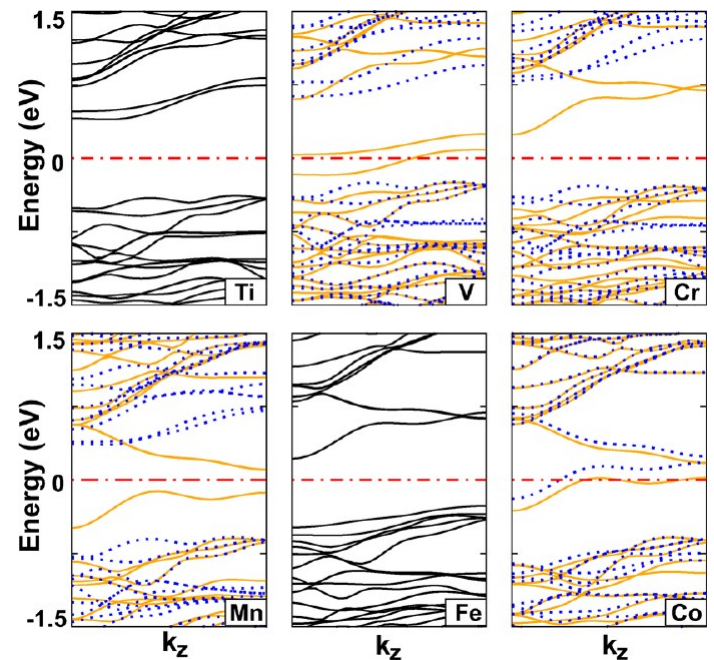

Figure 11. Electronic band structures of $\mathrm{H}-\mathrm{SiNW}(N)+\mathrm{TM}$ at site (5) for $N=45,57$, and 61 and $\mathrm{TM}=\mathrm{Ti}, \mathrm{V}, \mathrm{Cr}, \mathrm{Mn}, \mathrm{Fe}$, and $\mathrm{Co}$. Solid and dotted lines indicate spin up and down states, respectively.

systems. Moreover, GW corrections are performed on the frozen structure and do not allow for self-consistent structural relaxations.

Alternatively, calculations using hybrid functionals, ${ }^{59}$ which basically combine Hartree-Fock and DFT, usually provide improved electronic properties compared with DFT, although they are often less accurate for structural properties. Hybrid calculations are less costly than GW corrections and also allow for structural relaxations. Hybrid functionals were used to calculate the electronic structure of carbon nanotubes ${ }^{60-63}$ and graphene nanoribbons ${ }^{64}$ and yield good agreement with experimental data. More recently, Rurali et al. ${ }^{19}$ also applied hybrid functionals to silicon nanowires to determine accurately electronic band gap for varying diameter and orientation.

In this section, we compared the results obtained in the previous sections with those obtained by using B1-WC hybrid functional. We applied this technique only to specific cases, such as $\mathrm{H}-\mathrm{SiNW}(45)+\mathrm{Fe}$ at site (1), which is a ferromagnetic semimetal, $\mathrm{H}-\mathrm{SiNW}(45)+\mathrm{Fe}$ at site (5), which is a ferromagnetic semiconductor, and $\mathrm{H}-\mathrm{SiNW}(45)+\mathrm{Cr}$ (which is very similar to $\mathrm{H}-\mathrm{SiNW}(45)+\mathrm{Mn})$ at site (5), which is a halfmetal, and compare the results obtained with GGA and GGA $+\mathrm{U}^{37}$ calculations as shown in Figure 12.
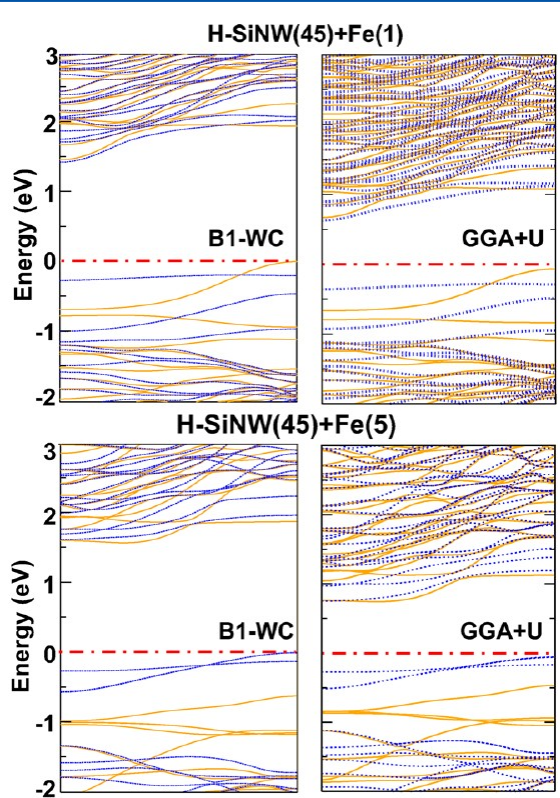

H-SiNW(45) $+\operatorname{Cr}(5)$
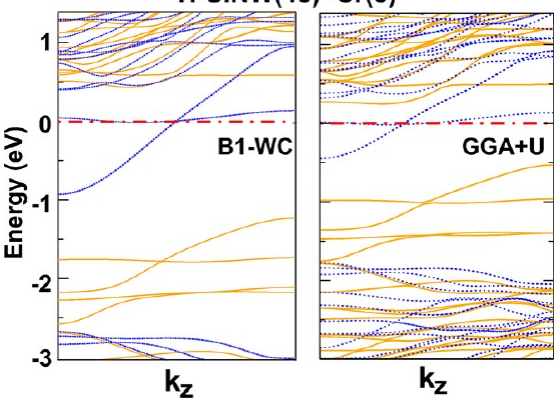

Figure 12. Electronic band structures of $\mathrm{H}-\mathrm{SiNW}(45)+\mathrm{Fe}$ at site (1), $\mathrm{HSiNW}(45)+\mathrm{Fe}$ at site (5), and $\mathrm{H}-\mathrm{SiNW}(45)+\mathrm{Cr}$ at site (5) obtained by B1-WC and GGA+U calculations. Solid and dotted lines indicate spin up and down states, respectively.

When B1-WC is used, the band structure of $\mathrm{H}$ $\mathrm{SiNW}(45)+\mathrm{Fe}$ at site (1) is altered and becomes semiconductor instead of semimetal. Similar band profile with a smaller band gap is also obtained when the Hubbard- $U(U=3$ $\mathrm{eV}$ ) term is considered for the strong on-site $3 \mathrm{~d}$ electronelectron interactions on Fe. Similar results are obtained for $\mathrm{H}$ $\mathrm{SiNW}(45)+\mathrm{Fe}$ at site (5). The system remains to be semiconducting, but band gap significantly increases when compared with GGA results. Accordingly, we conclude that semimetallic behavior of $\mathrm{H}-\mathrm{SiNW}(N)$ is an artifact of DFT at the GGA level and $\mathrm{H}-\mathrm{SiNW}$ remains semiconducting with a modified band gap upon Fe doping for both subsurface and core sites.

The novel half-metallic ground state of $\mathrm{H}-\mathrm{SiNW}(45)+\mathrm{Cr}$ at site (5) is preserved by B1-WC and also when the $U$ term is introduced. Interestingly, the dispersive metallic spin state $(\uparrow)$ is not affected by either approach, but the band gap for the insulating spin state $(\downarrow)$ is modified. In other words, halfmetallic ground states obtained at the DFT-GGA level ${ }^{20,21}$ are even more stable than predicted. 
Finally, the results indicate that B1-WC not only is capable of correcting the underestimated electronic band gaps but also can handle strong on-site $\mathrm{d}$ electron-electron interactions without introducing Hubbard- $U$ term, being a good alternative to $\mathrm{LDA}(\mathrm{GGA})+\mathrm{U}$ for strongly correlated systems ${ }^{65,66}$

\section{CONCLUSIONS}

We analyzed the structural, electronic, and magnetic properties of hydrogen-saturated silicon nanowires with interstitial as well as substitutional transition-metal doping. We found that the electronic and magnetic ground state is mainly determined by the type of (or d-electron configuration of) transition-metal atom, it can also be affected by doping type/site and cross section (surface effects) of silicon nanowire. Upon interstitial transition-metal doping, there is a tendency for metallization, except for the case of Fe. Even novel half-metallic configuration can be obtained for specific cases, and a different electronic structure profile can be obtained for substitutional doping. The stability of ferromagnetic ground state and energetics of binding are inversely proportional and depend on the number of unpaired 3d-electrons following the Friedel model. The obtained results are also compared with those calculated using a new type of hybrid functional (B1-WC) to clarify the limitations of DFT at GGA level and also to find out the capabilities of B1-WC. We believe that our detailed analysis will guide both experimental and theoretical studies related to doping of silicon nanowires and other systems; moreover, present results hold promise for the use of silicon nanowires in various spintronic applications upon transition-metal doping as a new type of dilute magnetic semiconductor.

\section{AUTHOR INFORMATION}

\section{Corresponding Author}

*E-mail: edurgun@mit.edu.

\section{Notes}

The authors declare no competing financial interest.

\section{ACKNOWLEDGMENTS}

This work was supported by the Interuniversity Attraction Poles Program (grant no. P6/42)-Belgian State-Belgian Science Policy, and the ARC project TheMoTher. Ph.G. acknowledges a Research Professorship from the Francqui Foundation. D.B. acknowledges additional financial support from the Romanian National Authority for Scientific Research, CNCS-UEFISCDI, project number PN-II-RU-TE-2011-3-0085.

\section{REFERENCES}

(1) Rurali, R. Rev. Mod. Phys. 2010, 82, 427-449.

(2) Hochbaum, A. I.; Yang, P. Chem. Rev. 2010, 110, 527-546.

(3) Wu, X.; Kulkarni, J. S.; Collins, G.; Petkov, N.; Almécija, D.; Boland, J. J.; Erts, D.; Holmes, J. D. Chem. Mater. 2008, 20, 59545967.

(4) Morales, A. M.; Lieber, C. M. Science 1998, 279, 208-211.

(5) Holmes, J. D.; Johnston, K. P.; Doty, R. C.; Korgel, B. A. Science 2000, 287, 1471-1473.

(6) Boukai, A. I.; Bunimovich, Y.; Tahir-Kheli, J.; Yu, J.-K.; Goddard, W. A., III; Heath, J. R. Nature 2008, 451, 168-171.

(7) Cui, Y.; Lauhon, L. J.; Gudiksen, M. S.; Wang, J.; Lieber, C. M. Appl. Phys. Lett. 2001, 78, 2214-2216.

(8) (a) Ma, D. D. D.; Lee, C. S.; Au, F. C. K.; Tong, S. Y.; Lee, S. T. Science 2003, 299, 1874-1877. (b) Zhang, R. Q.; Lifshitz, Y.; Ma, D. D. D.; Zhao, Y. L.; Frauenheim, T.; Lee, S. T.; Tong, S. Y. J. Chem. Phys. 2005, 123, 144703.

(9) Cui, Y.; Lieber, C. M. Science 2001, 291, 851-853.
(10) Lauhon, L. J.; Gudiksen, M. S.; Wang, D.; Lieber, C. M. Nature 2002, 420, 57-61.

(11) Cui, Y.; Zhong, Z.; Wang, D.; Wang, W. U.; Lieber, C. M. Nano Lett. 2003, 3, 149-152.

(12) Huang, Y.; Duan, X.; Lieber, C. Small 2005, 1, 142-147.

(13) Duan, X.; Huang, Y.; Agarwal, R.; Lieber, C. M. Nature 2003, 421, 241-245.

(14) Hahm, J.-i.; Lieber, C. M. Nano Lett. 2004, 4, 51-54.

(15) Zhao, X.; Wei, C. M.; Yang, L.; Chou, M. Y. Phys. Rev. Lett. 2004, 92, 236805.

(16) Vo, T.; Williamson, A. J.; Galli, G. Phys. Rev. B 2006, 74, 045116.

(17) Rurali, R.; Lorente, N. Phys. Rev. Lett. 2005, 94, 026805.

(18) Rurali, R; Poissier, A.; Lorente, N. Phys. Rev. B 2006, 74, 165324.

(19) Rurali, R.; Aradi, B.; Frauenheim, T.; Gali, A. Phys. Rev. B 2007, 76,113303

(20) Durgun, E.; Cakir, D.; Akman, N.; Ciraci, S. Phys. Rev. Lett. 2007, 99, 256806.

(21) Durgun, E.; Akman, N.; Ciraci, S. Phys. Rev. B 2008, 78, 195116.

(22) Cahangirov, S.; Ciraci, S. Phys. Rev. B 2009, 80, 075305.

(23) Akman, N.; Durgun, E.; Cahangirov, S.; Ciraci, S. Phys. Rev. B 2007, 76, 245427.

(24) Cui, Y.; Wei, Q.; Park, H.; Lieber, C. M. Science 2001, 293, $1289-1292$.

(25) Zhou, X.; Hu, J.; Li, C.; Ma, D.; Lee, C.; Lee, S. Chem. Phys. Lett. 2003, 369, 220-224.

(26) Singh, A. K.; Kumar, V.; Note, R.; Kawazoe, Y. Nano Lett. 2006, 6, 920-925.

(27) Fernández-Serra, M. V.; Adessi, C.; Blase, X. Phys. Rev. Lett. 2006, 96, 166805.

(28) Durgun, E.; Akman, N.; Ataca, C.; Ciraci, S. Phys. Rev. B 2007, $76,245323$.

(29) Wu, H. W.; Tsai, C. J.; Chen, L. J. Appl. Phys. Lett. 2007, 90, 043121.

(30) Giorgi, G.; Cartoixà, X.; Sgamellotti, A.; Rurali, R. Phys. Rev. B 2008, 78, 115327.

(31) Kohn, W.; Sham, L. J. Phys. Rev. 1965, 140, A1133-A1138.

(32) Kresse, G.; Hafner, J. Phys. Rev. B 1993, 47, 558-561.

(33) Kresse, G.; Joubert, D. Phys. Rev. B 1999, 59, 1758-1775.

(34) Blöchl, P. E. Phys. Rev. B 1994, 50, 17953-17979.

(35) Perdew, J. P.; Burke, K.; Ernzerhof, M. Phys. Rev. Lett. 1996, 77, 3865-3868.

(36) Monkhorst, H. J.; Pack, J. D. Phys. Rev. B 1976, 13, 5188-5192.

(37) Dudarev, S. L.; Botton, G. A.; Savrasov, S. Y.; Humphreys, C. J.; Sutton, A. P. Phys. Rev. B 1998, 57, 1505-1509.

(38) Heyd, J.; Scuseria, G. E.; Ernzerhof, M. J. Chem. Phys. 2006, 124, 219906.

(39) Bilc, D. I.; Orlando, R.; Shaltaf, R.; Rignanese, G.-M.; Íñiguez, J.; Ghosez, Ph. Phys. Rev. B 2008, 77, 165107.

(40) Wu, Z.; Cohen, R. E. Phys. Rev. B 2006, 73, 235116.

(41) Becke, A. D. J. Chem. Phys. 1996, 104, 1040-1046.

(42) Dovesi, R.; Orlando, R.; Civalleri, B.; Roetti, C.; Saunders, V. R.; Zicovich-Wilson, C. M. Z. Kristallogr. 2005, 220, 571-573.

(43) Peelaers, H.; Durgun, E.; Partoens, B.; Ghosez, Ph.; de Walle, C. G. V.; Peeters, F. M., 2012, unpublished.

(44) Porter, A. R.; Towler, M. D.; Needs, R. J. Phys. Rev. B 1999, 60, 13534-13546.

(45) Gatti, C.; Saunders, V. R.; Roetti, C. J. Chem. Phys. 1994, 101, $10686-10696$.

(46) Catti, M.; Sandrone, G.; Valerio, G.; Dovesi, R. J. Phys. Chem. Solids 1996, 57, 1735-1741.

(47) de P. R. Moreira, I.; Dovesi, R.; Roetti, C.; Saunders, V. R.; Orlando, R. Phys. Rev. B 2000, 62, 7816-7823.

(48) Cao, J. X.; Gong, X. G.; Zhong, J. X.; Wu, R. Q. Phys. Rev. Lett. 2006, 97, 136105.

(49) Ismail-Beigi, S.; Arias, T. Phys. Rev. B 1998, 57, 11923-11926.

(50) Martin, R. Electronic Structure Basic Theory and Practical Methods; Cambridge University Press: Cambridge, U.K., 2004. 
(51) Barone, V.; Hod, O.; Peralta, J. E.; Scuseria, G. E. Acc. Chem. Res. 2011, 44, 269-279.

(52) Heyd, J.; Peralta, J. E.; Scuseria, G. E.; Martin, R. L. J. Chem. Phys. 2005, 123, 174101.

(53) Ziman, J. M. The Physics of Metals; Friedel, J., Ed.; Cambridge University Press: New York, 1969.

(54) Durgun, E.; Dag, S.; Bagci, V. M. K.; Gülseren, O.; Yildirim, T.; Ciraci, S. Phys. Rev. B 2003, 67, 201401.

(55) Durgun, E.; Dag, S.; Ciraci, S.; Gülseren, O. J. Phys. Chem. B 2004, 108, 575-582.

(56) For the $\mathrm{H}-\mathrm{SiNW}(45)$ and impurity at site 5 (core), the computed binding energies for $\mathrm{Ti}, \mathrm{V}, \mathrm{Cr}, \mathrm{Mn}, \mathrm{Fe}$, and $\mathrm{Co}$ impurities are, respectively, 3.72 (3.83), 3.19 (3.32), 1.73 (1.83), 2.09 (2.17), 3.34 (3.70), and 3.85 (4.16) eV using a single (double) unit cell along the wire direction corresponding to distance between impurities of $\sim 5.4$ (10.8) Å.

(57) Hedin, L. Phys. Rev. 1965, 139, A796-A823.

(58) Bruno, M.; Palummo, M.; Marini, A.; Del Sole, R.; Ossicini, S. Phys. Rev. Lett. 2007, 98, 036807.

(59) Becke, A. D. J. Chem. Phys. 1993, 98, 1372-1377.

(60) Avramov, P. V.; Kudin, K. N.; Scuseria, G. E. Chem. Phys. Lett. 2003, 370, 597-601.

(61) Barone, V.; Peralta, J. E.; Uddin, J.; Scuseria, G. E. J. Chem. Phys. 2006, 124, 024709.

(62) Barone, V.; Peralta, J. E.; Wert, M.; Heyd, J.; Scuseria, G. E. Nano Lett. 2005, 5, 1621-1624.

(63) Barone, V.; Peralta, J. E.; Scuseria, G. E. Nano Lett. 2005, 5, $1830-1833$.

(64) Barone, V.; Hod, O.; Scuseria, G. E. Nano Lett. 2006, 6, 27482754 (PMID: 17163699).

(65) Goffinet, M.; Hermet, P.; Bilc, D. I.; Ghosez, Ph. Phys. Rev. B 2009, 79, 014403.

(66) Prikockyte, A.; Bilc, D.; Hermet, P.; Dubourdieu, C.; Ghosez, Ph. Phys. Rev. B 2011, 84, 214301. 